\title{
Spin Selective Charge Transport through Cysteine Capped CdSe Quantum Dots
}

Brian P. Bloom, ${ }^{\#}$ Vankayala Kiran, ${ }^{\#}$ Vaibhav Varade, Ron Naaman, David. H. Waldeck ${ }^{*}$

${ }^{\#}$ these authors contributed equally to this work

Department of Chemistry, University of Pittsburgh, Pittsburgh, Pennsylvania 15260

Department of Chemical Physics, Weizmann Institute, Rehovot 76100, Israel

Email: dave@pitt.edu

(412) 624-8430

\section{Supplementary Information}




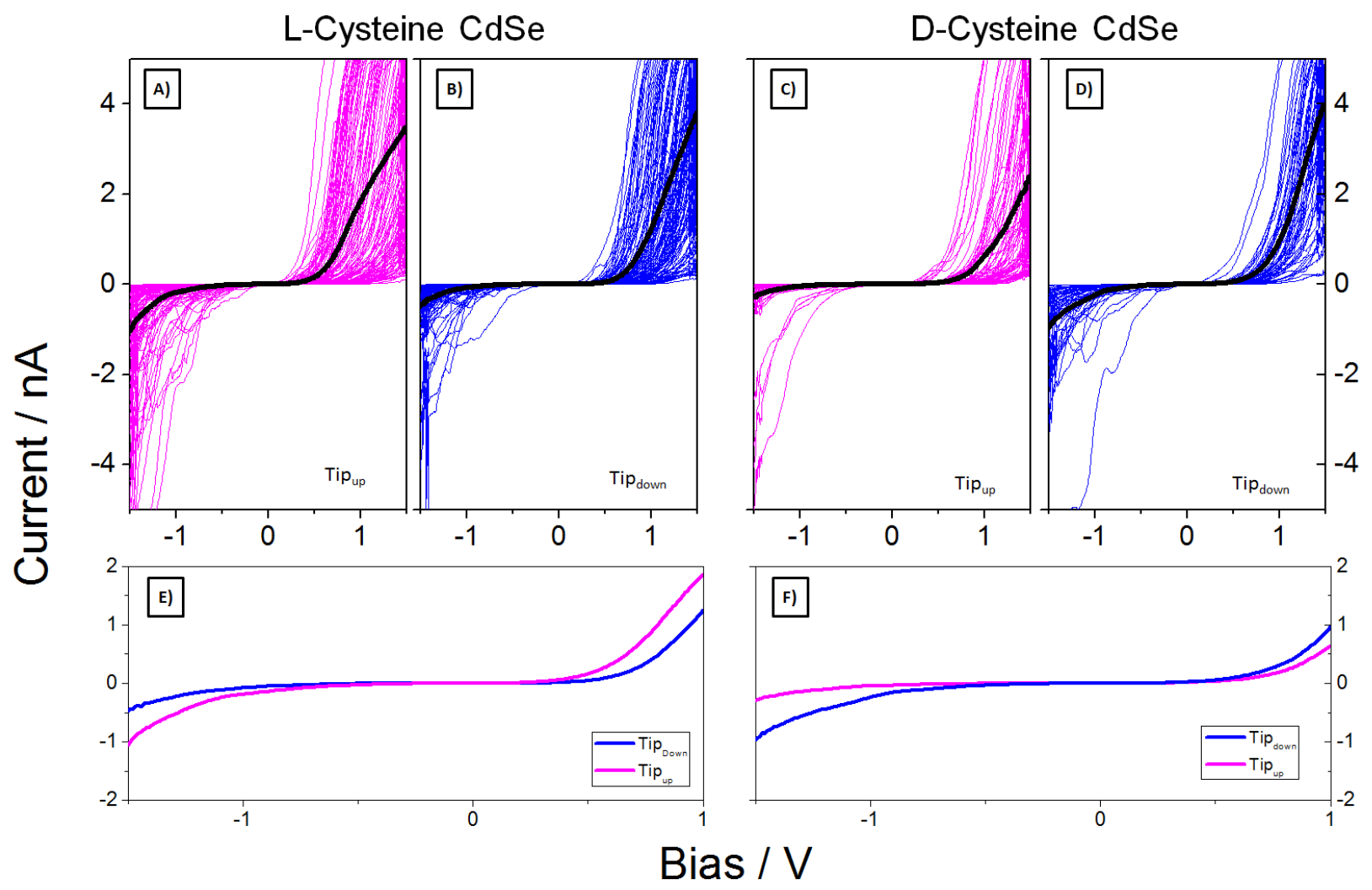

Figure S1. mCP-AFM studies of $2.2 \mathrm{~nm}$ L-cysteine $(\mathbf{A}, \mathbf{B}, \mathbf{E})$ and D-cysteine $(\mathbf{C}, \mathbf{D}, \mathbf{F})$ passivated CdSe QD films that are drop cast on HOPG substrates. The tip was magnetized in the up (magenta) and down (blue) direction and over 100 current-voltage curves were collected for each sample. The black line in (A-D) represents the average of all measurements for this data set. The effect of magnetization and chirality on the I-V curves are shown in $\mathbf{E}$ and F. For L-cysteine CdSe (D-cysteine CdSe) a higher current was observed when the tip was magnetized in the up (down) direction. 

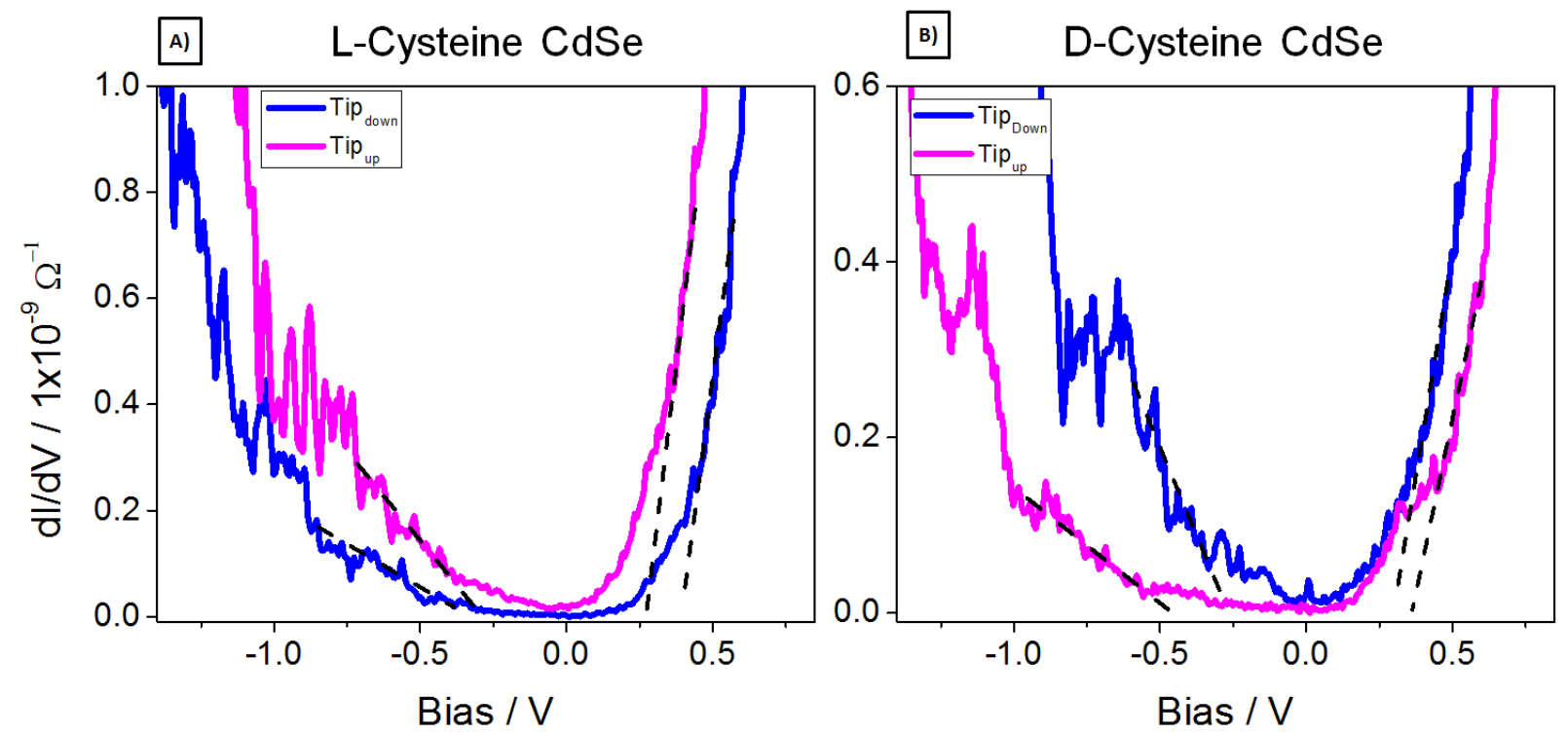

Figure S2. The dI/dV plots of the average mCP-AFM measurements for $2.2 \mathrm{~nm} \mathrm{L-}$ cysteine (A) and D-cysteine (B) passivated CdSe quantum dots. The black dashed lines correspond to the onset energy values for the two different electronic states; the conduction band at a positive bias and surface state defects at a negative bias. 

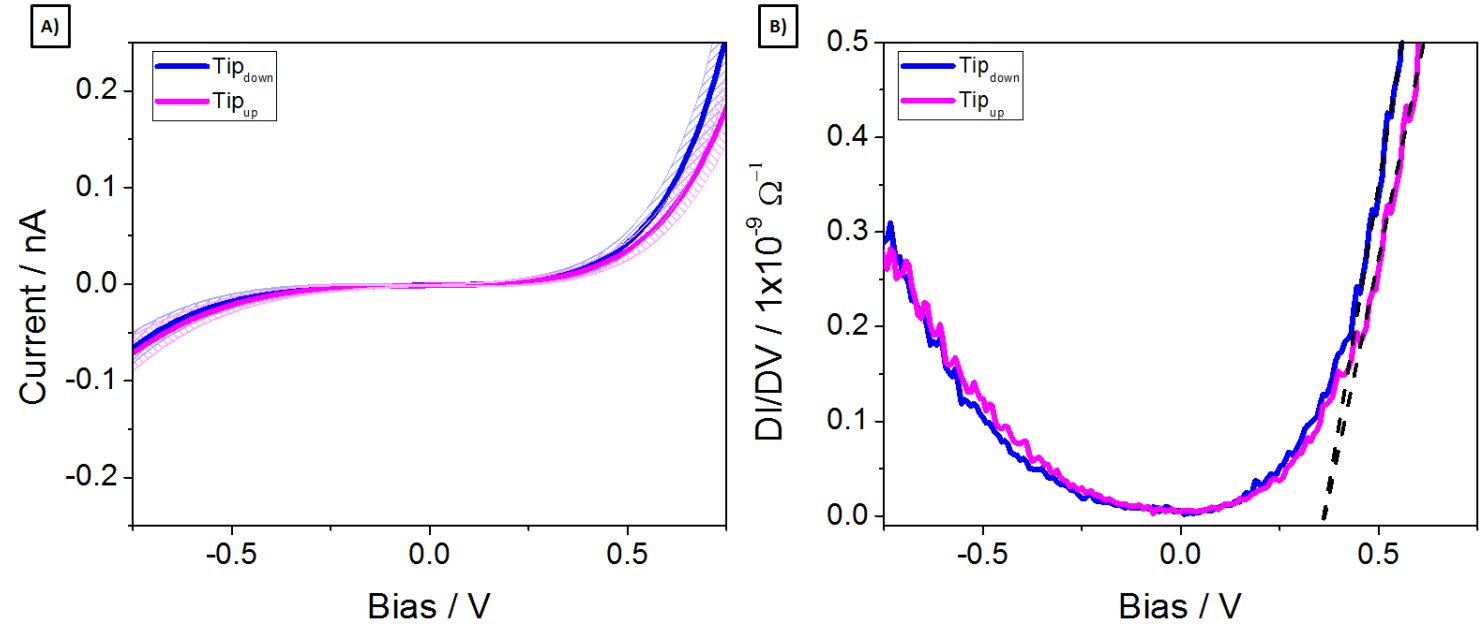

Figure S3. The current-voltage (A) and dI/dV (B) plots for $6.0 \mathrm{~nm}$ L-cysteine capped quantum dots drop cast on HOPG substrates. The black lines in panel B correspond to the onset region of the conduction band of the quantum dots. For this size of quantum dots very little difference in the conduction and density of states is observed when the tip is magnetized in the down (blue) and up (magenta) orientations. These data should be contrasted with Figures 2 and 3 for the 2.2 nm quantum dots. 


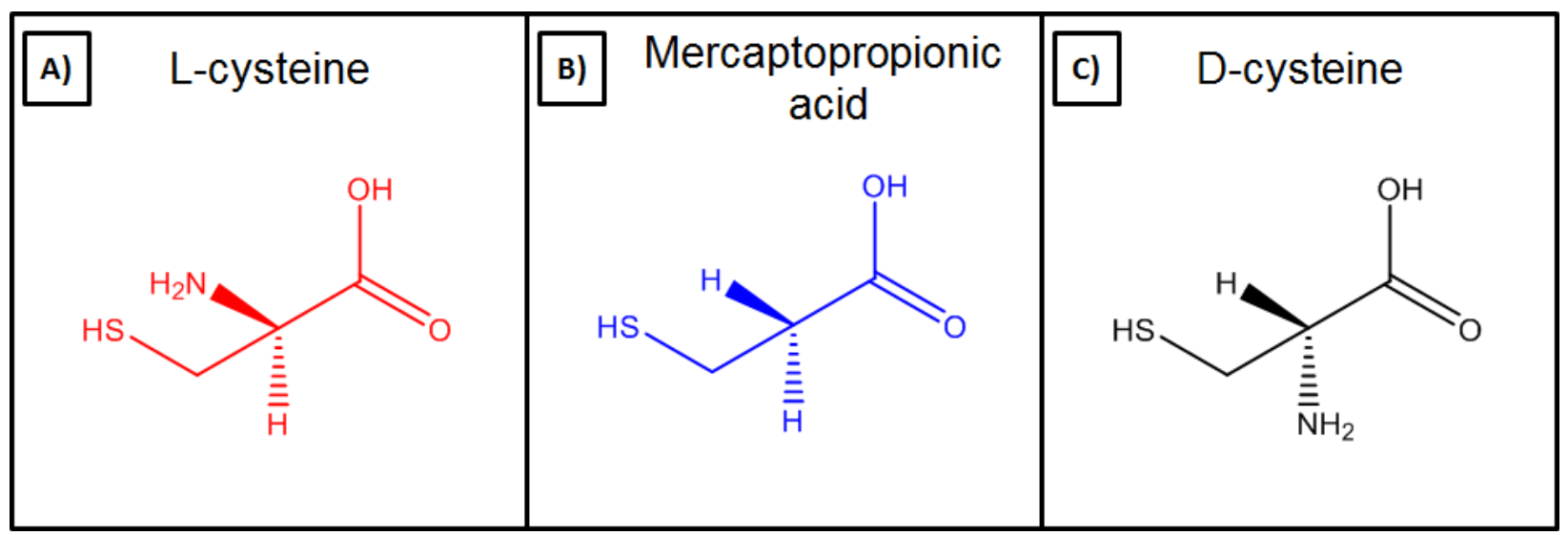

Figure S4. The molecular structures of the 3 ligands used in the magnetoresistance measurements; A) L-cysteine, B) 3-mercaptopropionic acid, and C) D-cysteine 


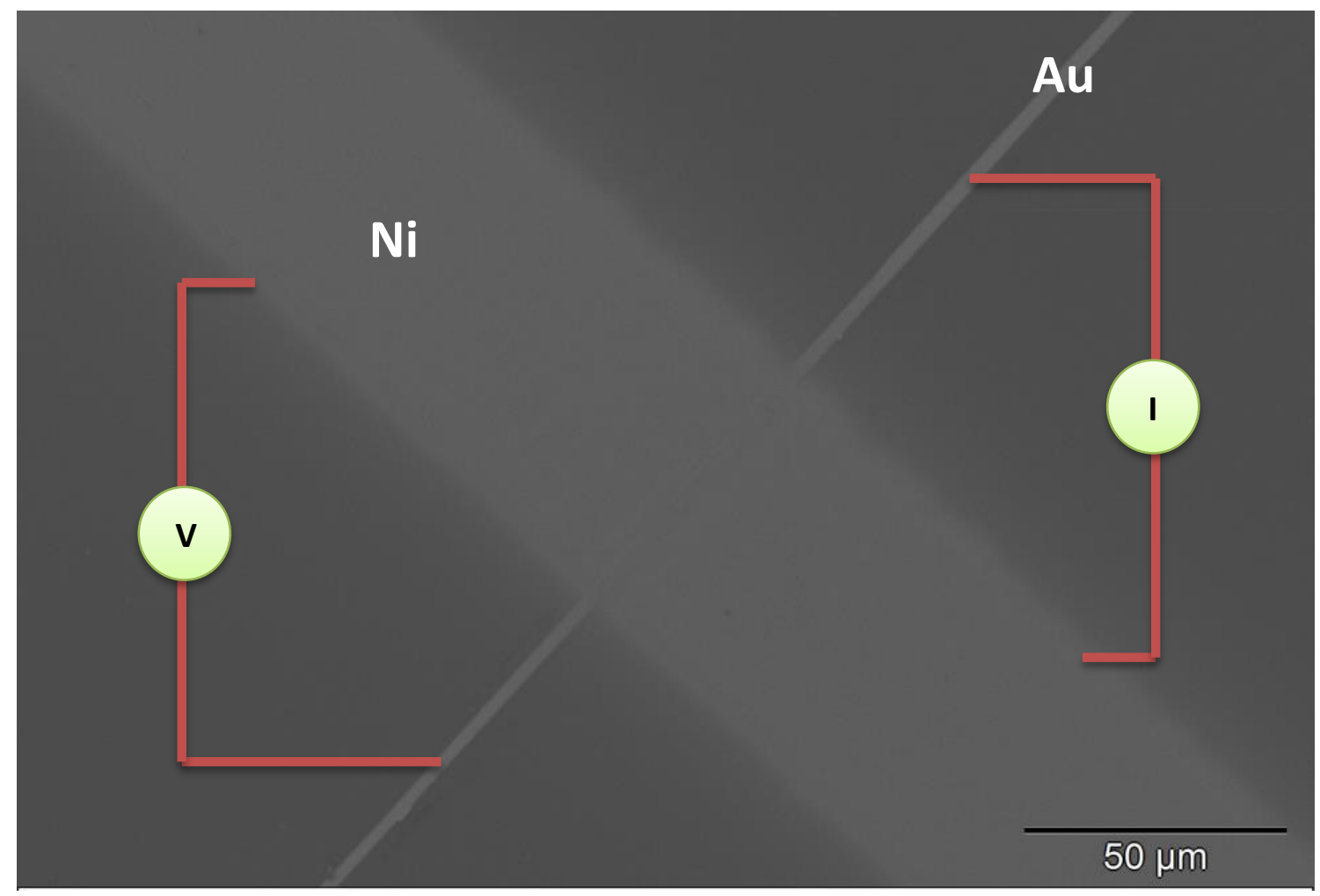

Figure S5: SEM image of the top view of the magnetoresistance device. 


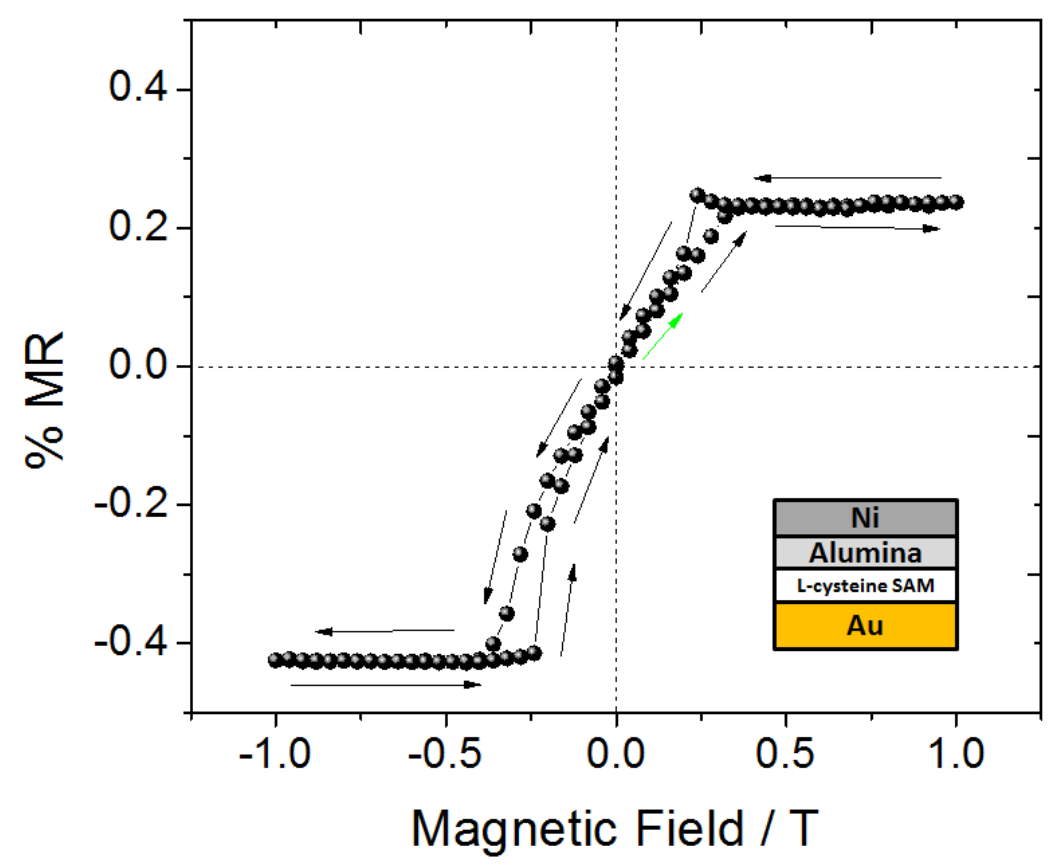

Figure S6. The magnetoresistance (MR) measured with a device which includes a L-cysteine SAM at $20 \mathrm{~K}$. It is important to note that the orientation of the asymmetric response is opposite to that of the devices made with L-cysteine coated CdSe nanoparticles. 

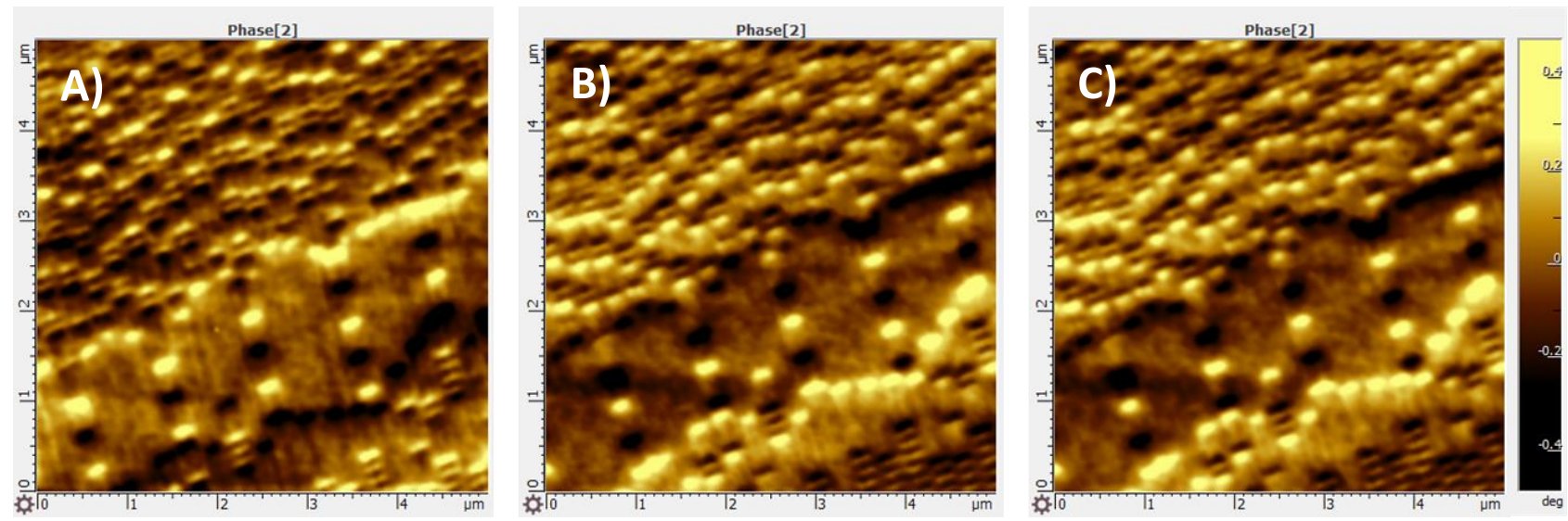

Figure S7. Shows MFM images recorded using a Co-Cr tip magnetized in (A) DOWN, (B) UP and (C) UP after 2 hours. The domains in (A) and (B) are opposite to each other, confirming the reversal of magnetic polarity of the tip between DOWN and UP orientations. The fidelity of the two MFM images (middle \& right) shows that the tip does not undergo oxidation or demagnetization over the course of the 2 hours. 

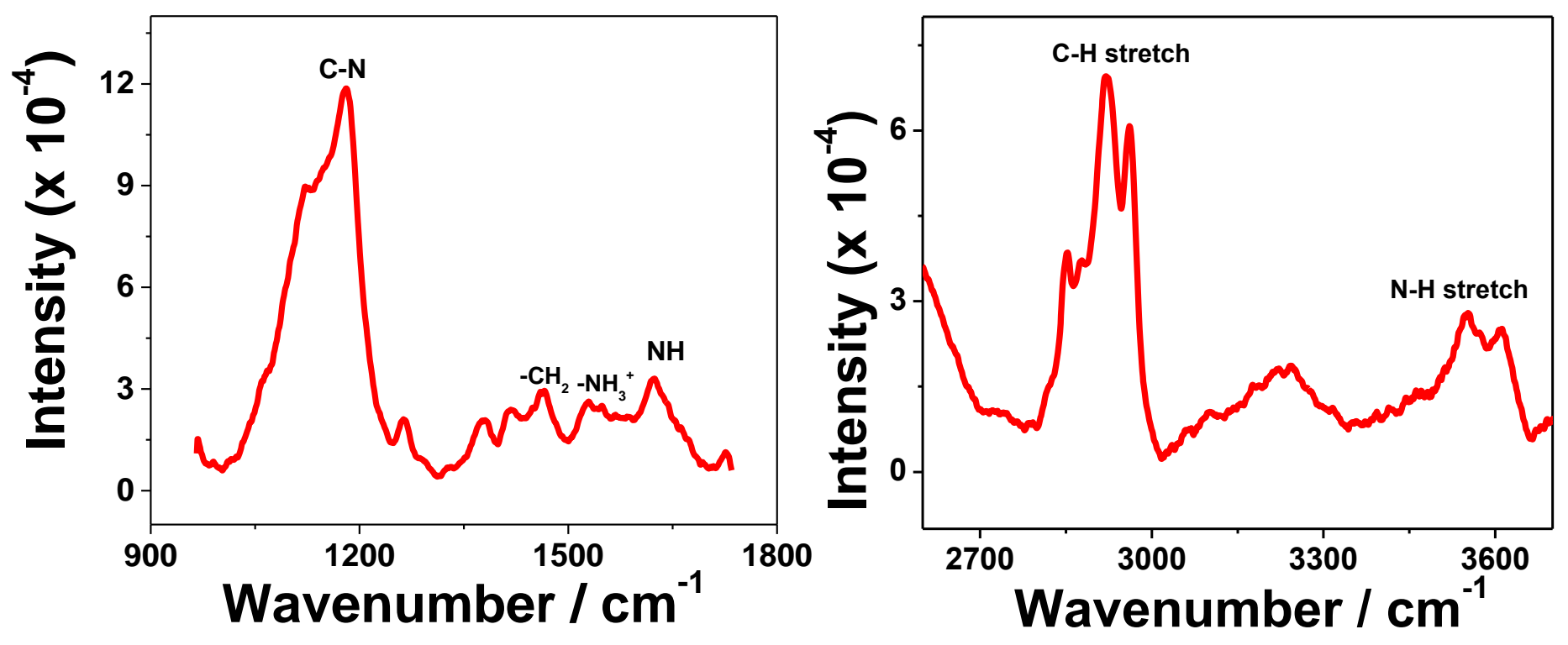

Figure S8. PMIRRAS of 2-aminoethanethiol monolayers adsorbed on $\mathrm{Au}$ recorded in two different regions.

Adsorption of the self-assembled monolayer (cysteamine) used in the MR devices were confirmed using PMIRRAS spectra figureS7. The spectra's peaks are labled C-N, N-H and C-H corresponding to the different stretches of the cysteamine monolayer. The peaks located at 1175 $\mathrm{cm}^{-1}$ and $1625 \mathrm{~cm}^{-1}$ correspond to the $\mathrm{C}-\mathrm{N}$ stretch and the deformation asymmetric vibration ($\mathrm{NH}$ ) of primary ammonium functions respectively. The peaks located around $3400-3600 \mathrm{~cm}^{-1}$ represents $\mathrm{N}-\mathrm{H}$ stretching modes of the amine group. Also, the vibrations corresponding to $\mathrm{C}-\mathrm{H}$ modes are also seen in the spectra around $2800-3000 \mathrm{~cm}^{-1}$. 


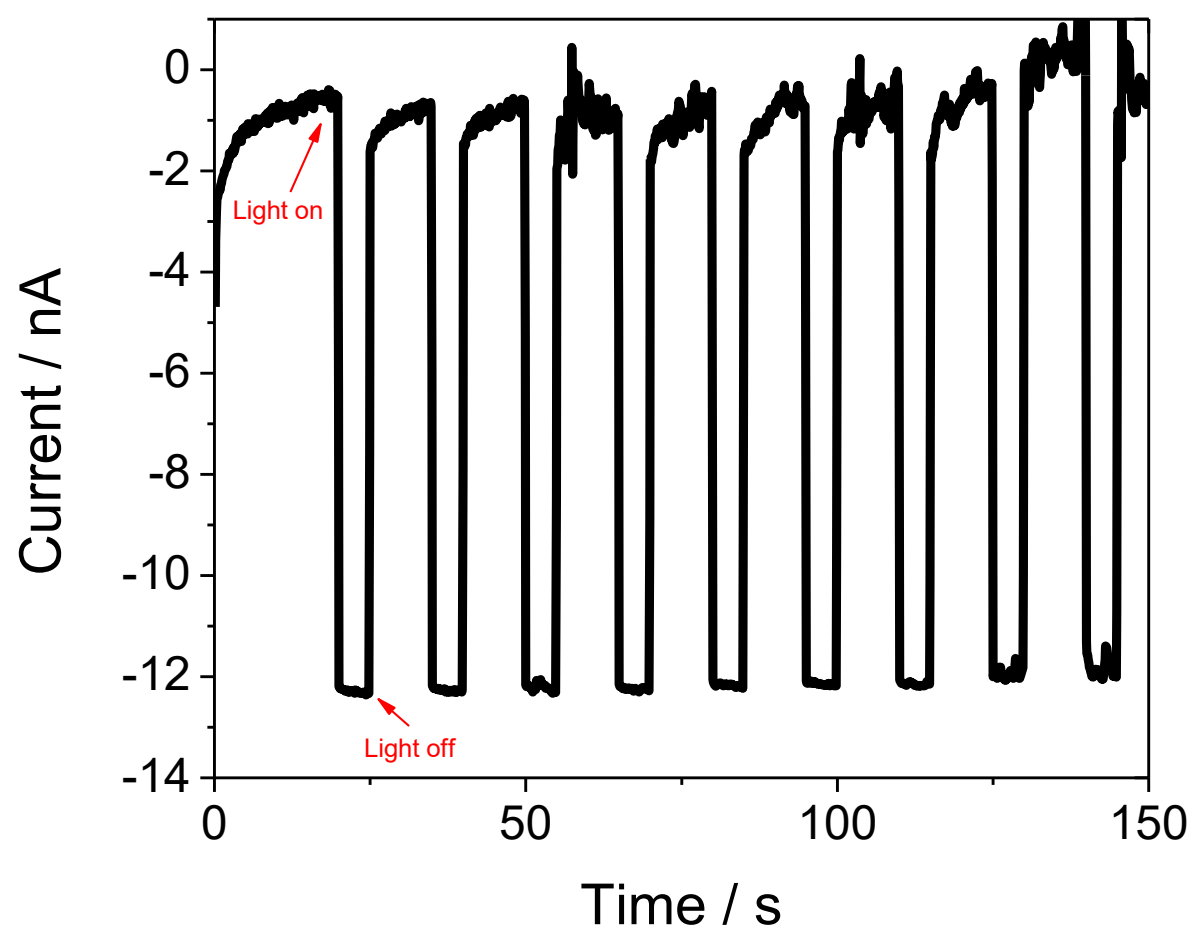

Figure S9. Shows photocurrent measurements made on L-cys CdSe QDs electrostatically attached to an $\mathrm{Au}$ electrode - 8-mercaptooctylamine SAM. The counter electrode was a platinum mesh and the auxiliary electrode was $\mathrm{Ag} / \mathrm{AgCl}$ (CHInstruments). The supporting electrolyte was a $0.1 \mathrm{M} \mathrm{NaS}$ solution and measurements were performed under a $0 \mathrm{~V}$ bias potential. 